\title{
Matching Delaunay Graphs
}

\author{
Andrew M. Finch and Edwin R. Hancock \\ Department of Computer Science, \\ University of York, U.K.
}

\begin{abstract}
This paper describes a Bayesian framework for matching Delaunay graphs. Our matching process is realised in terms of probabilistic relaxation. The novelty of our method stems from its use of a support function specified in terms of triangular face-units of the graphs under match. In this way we draw on more expressive constraints than is possible at the level of edge-units alone. We present a particularly simple face compatibility model that is entirely devoid of free parameters. It requires only knowledge of the numbers of nodes, edges and faces in the model graph. The resulting matching scheme is evaluated on radar images and compared with its edge-based counterpart.
\end{abstract}

\section{Introduction}

Inexact graph matching is a critical process for effective intermediate level scene interpretation $[2,4,5,6]$. The technique allows a corrupted relational description of noisy image entities to be matched against an idealised model graph. As a consequence of its pivotal role in practical vision applications, the graph matching problem has been the focus of sustained activity in a number of diverse methodological areas. Early efforts focussed on the search for subgraph isomorphisms [5] and the definition of a meaningful relational distance metric [5]. More recently, optimisation ideas have provided a powerful alternative framework for relational matching $[2,4,6]$.

Basic to these inexact methods is the aim of optimising a measure of matching consistency [4]. Ideally, this measure should be capable of simultaneously capturing both the constraints conveyed by the model graph and the corruption processes at play in the data graph. The majority of matching algorithms are satisfied with exploiting only the pairwise constraints provided by graph edges [4]. This restricted use of available relational constraints not only limits the achievable performance, it may also actually lead to internal inconsistencies in the specification of the matching process. In effect it corresponds to discarding a considerable body of detailed information concerning the local topology of the relational graphs under match.

Delaunay graphs provide an important example where the relational structure is not adequately represented at the edge-level [1]. These graphs are composed of triangular faces and arise in the representation of Voronoi tesselations of planes or surfaces. The intrinsic relational structure of Delaunay graphs is invariably overlooked when it comes to matching. Our aim in this paper is to develop an internally consistent matching process for Delaunay graphs by drawing directly upon their local triangulated topology. In so doing we aim to exploit 
more of the available relational structure and hence improve the fidelity of match beyond that achievable with edge-based constraints alone [4]. Our framework for this study is provided by probabilistic relaxation. Application of this optimisation approach to the matching problem, requires that we develop not only a support-function that draws on triangulated graph units, but also an associated model of compatibility expressed at the level of consistent graph faces. Both of these ingredients are prescribed in terms of a Bayesian framework.

\section{Probabilistic Relaxation}

We denote Delaunay graphs by the triple $\mathcal{G}=(\mathcal{V}, \mathcal{E}, \mathcal{F})$. Here $\mathcal{V}$ is the set of nodes and $\mathcal{E} \subset \mathcal{V} \times \mathcal{V}$ is the set of edges. The novel ingredient of the work reported here is to exploit constraints provided by the set of triangular faces $\mathcal{F}$ in the matching process. We use the term face to mean a Cartesian triple of of node labels, i.e. $\mathcal{F} \subset \mathcal{V} \times \mathcal{V} \times \mathcal{V}$, such that $(i, j, k) \in \mathcal{F} \Leftrightarrow(i, j) \in \mathcal{E} \wedge(j, k) \in \mathcal{E} \wedge(k, i) \in \mathcal{E}$.

Previous work on relational matching has concentrated on exploiting constraints provided by the edge-set $\mathcal{E}_{m}$ and is not well suited to the matching of Delaunay graphs. It has been shown that edge-based strategies are best suited to matching graphs which are tree-like in form (i.e. graphs of low edge density in which $|\mathcal{V}| \approx|\mathcal{E}|$ ) containing few first order cycles (triangular faces). In this paper we aim to overcome this shortcoming by developing a matching scheme which draws on constraints represented by the face-sets of the data and model graphs. In so doing, we aim take advantage of the powerful constraints provided by the cyclic ordering of the nodes in the graphs under match. This ordering concept is central to the ideas presented here and is illustrated in Fig 1. Such an ordering exists if the graph is planar.

We define a graph $\mathcal{G}_{d}$ on the labels $J_{\alpha}$ of the objects $u_{\alpha}, \alpha=1 \ldots N$ to be: $\mathcal{G}_{d}=\left(\mathcal{V}_{d}, \mathcal{E}_{d}, \mathcal{F}_{d}\right)$, where $\mathcal{V}_{d}=\left\{J_{1}, \ldots, J_{N}\right\}$. The graph $G_{m}=\left(\mathcal{V}_{m}, \mathcal{E}_{m}, \mathcal{F}_{m}\right)$, where $\mathcal{V}_{m}=\left\{j_{1}, \ldots, j_{m}\right\}$ is defined on the values $j_{\alpha}$ attributable to these labels. In order to accommodate entries in the data graph that have no physical match in the model graph, we augment the indexed set of data nodes $\mathcal{V}_{m}$ by a null category $\phi$. This symbol us used to label unmatchable data entities which represent noise or extraneous clutter. We denote the index set of the contextual neighbours of

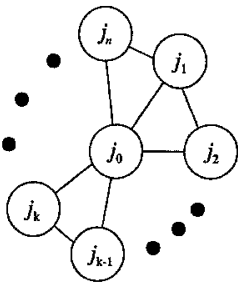

Fig. 1. Label value $j_{0}$ together with its contextual neighbours $j_{1} \ldots j_{n}$ object $u_{\alpha}$ as $I_{\alpha}$. This set includes the index $\alpha$. For the purpose of notational simplicity and without loss of generality, we re-index the objects in the network. This ensures that the object under consideration has index 0 and its neighbours are indexed from $1 \ldots n$ (as in Fig. 1).

The starting point for developing the matching strategy is the general purpose Bayesian evidence combining formula of Kittler and Hancock [3]. According to 
this framework, the probability at iteration $i$ for the match $J_{0} \mapsto j_{0}$ on the centrenode of the contextual neighbourhood is updated as follows:-

$$
P^{(i+1)}\left(J_{0} \mapsto j_{0}\right) \leftarrow \frac{P^{(i)}\left(J_{0} \mapsto j_{0}\right) Q^{(i)}\left(J_{0} \mapsto j_{0}\right)}{\sum_{j_{\alpha} \in \mathcal{V}_{m} \cup \phi} P^{(i)}\left(J_{0} \mapsto j_{\alpha}\right) Q^{(i)}\left(J_{0} \mapsto j_{\alpha}\right)}
$$

The critical ingredient in this formula is the support function $Q^{(i)}\left(J_{0} \mapsto j_{0}\right)$ which combines evidence for the match $J_{0} \mapsto j_{0}$ over the reindexed contextual neighbourhood of object $u_{0}$. For the triangulated neighbourhood shown in Fig 1 the support function may be evaluated recursively over the constituent faces faces commencing at the node indexed 1:-

$$
\begin{aligned}
Q^{(i)}\left(J_{0} \mapsto j_{\gamma}\right)= & \frac{1}{P\left(J_{0} \mapsto j_{\gamma}\right)} \sum_{j_{1} \in \mathcal{V}_{m} \cup \phi} \frac{P^{(i)}\left(J_{1} \mapsto j_{1}\right)}{P\left(J_{1} \mapsto j_{1}\right)} \\
& \times \sum_{j_{n} \in \mathcal{V}_{m} \cup \phi} P\left(J_{1} \mapsto j_{1} \mid J_{n} \mapsto j_{n}, J_{0} \mapsto j_{0}\right) \epsilon\left(J_{1} \mapsto j_{1}, J_{n} \mapsto j_{n}\right)
\end{aligned}
$$

We initialise this recursion by setting:-

$$
\epsilon\left(J_{n-1} \mapsto j_{n-1}, J_{n} \mapsto j_{n}\right)=\frac{P^{(i)}\left(J_{n} \mapsto j_{n}\right)}{P\left(J_{n} \mapsto j_{n}\right)} P\left(J_{n} \mapsto j_{n} \mid J_{n-1} \mapsto j_{n-1}, J_{0} \mapsto j_{0}\right)
$$

The basic recursion-kernel employed in evaluating support is:-

$$
\begin{aligned}
\epsilon\left(J_{k-1} \mapsto j_{k-1}, J_{n} \mapsto j_{n}\right) & =\sum_{j_{k} \in \mathcal{V}_{m} \cup \phi} \frac{P^{(i)}\left(J_{k} \mapsto j_{k}\right)}{P\left(J_{k} \mapsto j_{k}\right)} \\
& \times P\left(J_{k} \mapsto j_{k} \mid J_{k-1} \mapsto j_{k-1}, J_{0} \mapsto j_{0}\right) \epsilon\left(J_{k} \mapsto j_{k}, J_{n} \mapsto j_{n}\right)
\end{aligned}
$$

In the recursion formula (4), the index $J_{k} \mapsto j_{k}$ of the quantity $\epsilon\left(J_{k} \mapsto j_{k}, J_{n} \mapsto j_{n}\right)$ is needed because the label assigned to each object enters two levels of summation in a cyclic manner; the index $J_{n} \mapsto j_{n}$ is required since it is necessary to break the cyclicity of indices at some object in the neighbourhood, in order to perform a recursive evaluation. This twofold level of summation effectively propagates the face-based constraints around the perifery of the Delaunay neighbourhood. The Bayesian ingredients of the support function are the single node prior $P\left(J_{k} \mapsto j_{k}\right)$ and the conditional prior $P\left(J_{k} \mapsto j_{k} \mid J_{k-1} \mapsto j_{k-1}, J_{0} \mapsto j_{0}\right)$. It is the conditional prior that measures the consistency between the label match $J_{k} \mapsto j_{k}$ and the matches $J_{k-1} \mapsto j_{k-1}$ and $J_{0} \mapsto j_{0}$ on the remaining nodes of the face $\left\{J_{k}, J_{k-1}, J_{0}\right\}$ belonging to the data graph. According to our Bayesian framework, the mutual information measure $R\left(j_{k}, j_{k-1}, j_{0}\right)=\frac{P\left(J_{k} \mapsto j_{k} \mid J_{k-1} \mapsto j_{k-1}, J_{0} \mapsto j_{0}\right)}{P\left(J_{k} \mapsto j_{k}\right)}$ may be viewed a plying the role of a compatibility coefficient. In constructing a model of the compatibility coeffcients $R\left(j_{k}, j_{k-1}, j_{0}\right)$ we would like to capture some of the constraint violations introduced by different classes of segmentation error. These errors include noise contamination, fragmentation due to over segmentation and accidental merging due to under segmentation. 


\section{Compatibility Model}

Our adopted modelling philosophy is that the nodes in graph $\mathcal{G}_{d}$ represent data that must be matched in the light of constraints provided by graph $\mathcal{G}_{m}$. In order to cope with inconsistencies between the model and data graphs, we employ a system of constraint softening in which it is assumed that segmentation errors occur with uniform probability $p$. There are four classes of constraint corruption that can occur at the face level. Firstly, faces of the data graph with all nodes matched to a face in the model graph are uncorrupted and occur with total probability mass $(1-p)^{3}$. Secondly, there are faces with two nodes matched to an an edge in the model graph and one-node matched to the null label; these have total probability mass $p(1-p)^{2}$. The third class consists of faces in which two nodes are null-matched have a probability of $p^{2}(1-p)$. Finally, there are configurations in which all nodes in the face are null matched take the remaining probability mass, i.e. $p^{3}$. Matches involving non-null label triplets which are inconsistent with the above configurations are completely forbidden and therefore account for zero total probability mass. In each of the four cases listed above, the available mass of probability is distributed uniformly among the label configurations falling into the relevant constraint class. Using this we arrive at a rule which yields the joint probabilities for face configurations:-

$$
P\left(J_{k} \mapsto j_{k}, J_{k-1} \mapsto j_{k-1}, J_{0} \mapsto j_{0}\right)= \begin{cases}\frac{(1-p)^{3}}{\left|\mathcal{F}_{m}\right|} & \text { if }\left\{j_{k}, j_{k-1}, j_{0}\right\} \in \mathcal{F}_{m} \\ \frac{p(1-p)^{2}}{\left|\mathcal{E}_{m}\right|} & \text { if }\left\{j_{k}, j_{k-1}\right\} \in \mathcal{E}_{m} \wedge j_{0}=\phi \\ \frac{p^{2}(1-p)}{\left|\mathcal{V}_{m}\right|} & \text { if } j_{k} \in \mathcal{V}_{m} \wedge j_{k-1}=\phi \wedge j_{0}=\phi \\ p^{3} & \text { if } j_{k}=\phi \wedge j_{k-1}=\phi \wedge j_{0}=\phi \\ 0 & \text { otherwise }\end{cases}
$$

The edge-priors and single-node priors required in the computation of compatibility coefficients are obtained by summing the joint probabilities in the axiomatic way. The resulting compatibility coefficients are given below:-

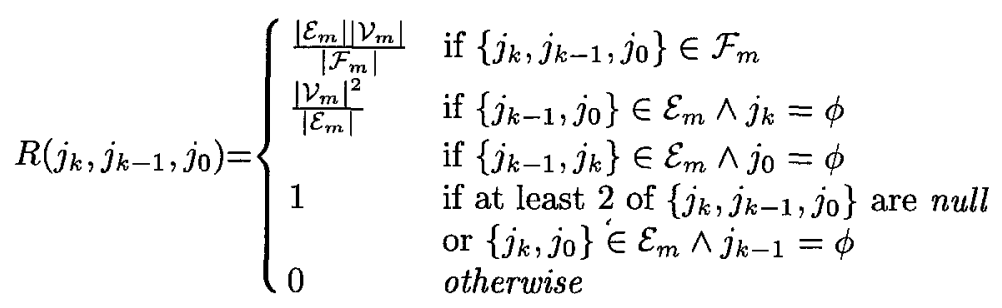

The pattern of compatibilities grades the different face-constraints according to their overall consistency. Fully consistent faces have higher compatibility values than partially matched faces which feature isolated edges or nodes. These compatibilities also discourage the violation of the neighbourhood ordering relation. Partially matched faces containing an edge which could could potentially disrupt the ordering of the contextual neighbourhood (i.e. $\left\{j_{k}, j_{0}\right\} \in \mathcal{E}_{m}$ ) receive a lower compatibility than the others which could not. In other words, 
the compatibility pattern favours null matched nodes that are surrounded by a consistently ordered neighbourhood over matches that are connected to a plethora of incorrectly ordered yet consistent edges. This ability to impose ordering relations enhances the internal consistency of our face-based relaxation scheme and represents the main advantage over edge-based compatibility models [4].

\section{Experiments}

In order to demonstrate some of the performance advantages of our face-based matching scheme, we have taken an application involving synthetic aperture radar data. We are interested in matching linear hedge structures from radar images against their cartographic representation in a digital map. We establish Delaunay graph representations of data and model, by seeding Voronoi tessellations from the midpoints of the linear segments. To illustrate the results of applying our face-based matching process to the Delaunay graphs, Fig 2 shows the correctly matched segments overlaid on the original radar image.
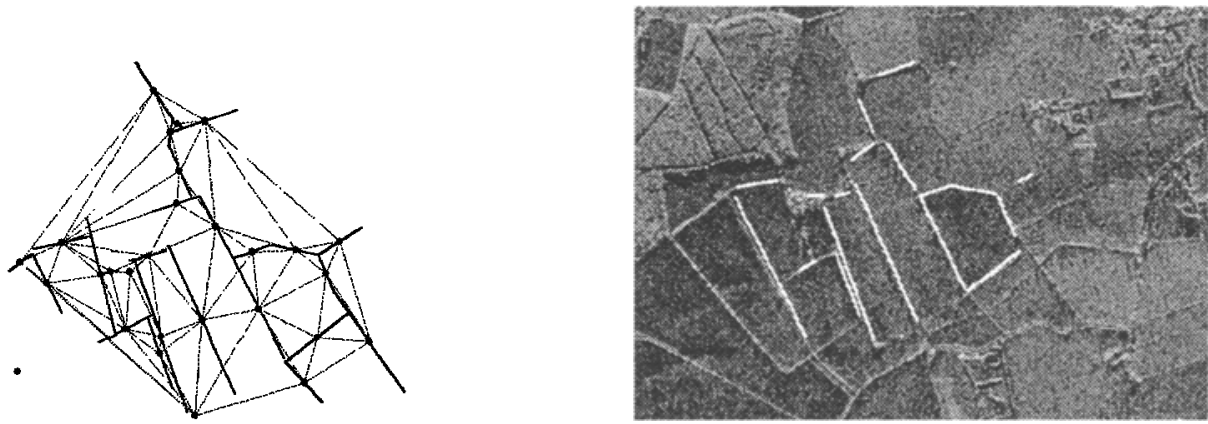

Fig. 2. The model graph (left) and correctly matched SAR line segments (right)

To give some idea of the effectiveness of our compatibility model, there 28 nodes, 46 faces and 142 edges in the model graph. In consequence, the face compatibility $\frac{\left|\mathcal{E}_{m}\right|\left|\mathcal{V}_{m}\right|}{\left|\mathcal{F}_{m}\right|}=86.43$, while the edge compatibility $\frac{\left|\mathcal{V}_{m}\right|^{2}}{\mathcal{E}_{m}}=5.52$. The graph in Fig 3 shows the accuracy of match (defined here to be the number of correctly matched line segments, minus the number of incorrectly matched line segments) plotted against the edge and face compatibility values. The graph shows a broad plateau around the theoretically optimal parameter values. In this region (3-8 edge compatibility and 30-100 face-compatibility) the correct/incorrect surplus is consistently high (i.e. 7-9). This is an encouraging observation, it means that our very simple compatibility model is adequate in describing the matching errors in a complex experimental example without excessive parameter tuning. Moreover, the results obtained with the face-based technique offer a number of tangible advantages over their edge-based counterpart. In the case of the facebased method 18 nodes are correct, 64 are null matched and only 5 nodes match 
in error. For the edge-based method, on the other hand, although 19 nodes are correctly matched, only 22 are null matched with 46 residual nodes matched in error. In other words, the face-based method is more effective in identifying unmatchable elements and assigning them to the null category.

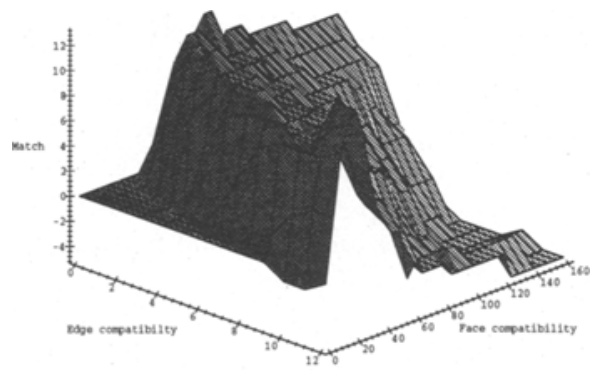

Fig. 3. Matching accuracy vs. face and edge compatibility

\section{Conclusions}

We have described a novel Bayesian framework for matching Delaunay triangulations. Our matching process is realised in terms of probabilistic relaxation. The novelty of our method stems from its use of a support function specified in terms of face-units of the graphs under match. In this way we draw on more expressive constraints than is possible at the level of edge-units alone. Viewed from the perspective of information processing utility, our method represents two basic advances over edge-based matching schemes. Firstly, we draw on the use of more powerful constraints since we are able to take advantage of neighbourhood ordering relations. Secondly, we are capable of matching graphs of high edge density containing many first-order cycles.

\section{References}

1. N. Ahuja, B. An and B. Schachter, "Image Representation using Voronoi Tessellation", CVGIP, 29, pp 286-295, 1985.

2. K. Boyer and A. Kak, "Structural Stereopsis for 3D Vision", IEEE PAMI, 10, pp 144-166, 1988.

3. J. Kittler and E.R. Hancock, "Contextual Decision Rule for Image Analysis", Image Vision Computing, 5, pp, 145-154, 1987.

4. J. Kittler, W.J. Christmas and M. Petrou, "Probabilistic Relaxation for Matching Problems in Machine Vision", Proceedings of the Fourth International Conference on Computer Vision, pp. 666-674, 1993.

5. L. Shapiro and R.M. Haralick, "Structural Description and Inexact Matching", IEEE PAMI, 3, pp 504-519, 1981.

6. R.C. Wilson and E.R Hancock, "Graph Matching by Discrete Relaxation", Pattern Recognition in Practice IV: Multiple Paradigms, Comparative Studies and Hybrid Systems, North Holland, pp. 165-177, 1994. 